\title{
An experimental and numerical study on vascular self- healing cementitious materials
}

\author{
Anthony Jefferson ${ }^{1 *}$, Tharmesh Selvarajoo ${ }^{1}$, Brubeck Freeman ${ }^{1}$, Robert Davies ${ }^{1}$, \\ ${ }^{1}$ Cardiff University, School of Engineering, UK
}

\begin{abstract}
This paper gives an overview of a combined experimental-numerical study on vascular self-healing $(\mathrm{SH})$ systems for cementitious composite materials. The work aimed to bridge the gap between numerical and experimental investigations for this type of SH system and to provide a set of data for developing, calibrating and validating a finite element model for these materials. The study investigated both healing-agent transport and mechanical damage-healing processes, including healing-agent curing. The experimental programme included mechanical tests on notched concrete beams and compact direct-tension specimens with inbuilt vascular healing systems, as well as tests to measure the transport properties of healing-agent within discrete concrete cracks and through the concrete matrix. The new coupled model employs elements with embedded strong discontinuities to simulate cracks and mechanical healing behaviour. A damage-healing constitutive model is described that simulates multiple damage-healing 'events'. This mechanical model is coupled to discrete and continuum flow models that simulate healing-agent transport. The transport model accounts for pressurised and capillary flow, as well as curing-dependent flow properties. The main focus of this contribution is to show how these parallel programmes of work were combined so that the experimental observations guided the numerical developments and modelling questions were answered using experimental findings.
\end{abstract}

\section{Introduction}

Interest in self-healing concrete has grown considerably in recent years due to the potential of these materials to greatly improve the durability properties of concrete structures [1]. Much of the work undertaken on these materials has been experimental in nature but there has also been some numerical research aimed at producing computational models for these materials [2]. Whilst considerable progress has been made on models that simulate certain aspects of self-healing behaviour, there remain some key behavioural characteristics that current models do not represent well, which includes the simulation of simultaneous and multiple damage-healing 'events'. A further problem with much previous numerical work is that the models have been developed, calibrated and validated using little, or sometimes no, experimental work [2]. To remedy this situation, a combined experimental-numerical study has been undertaken at Cardiff University on a particular vascular self-healing system. The present paper gives an outline description of this work. A full description will be provided in a series of forthcoming publications.

\section{Experimental Programme}

The experimental programme of work focussed on the behaviour of a vascular self-healing system, which has healing-agent supply channels embedded in cementitious structural elements. The healing agent used was cyanoacrylate (PC20).

The various test series undertaken are summarised below.

(i) Notched fracture beam tests with a range of a) fixed healing periods, b) loading rates and c) healingagent supply pressures (See Figs. 1 and 8).

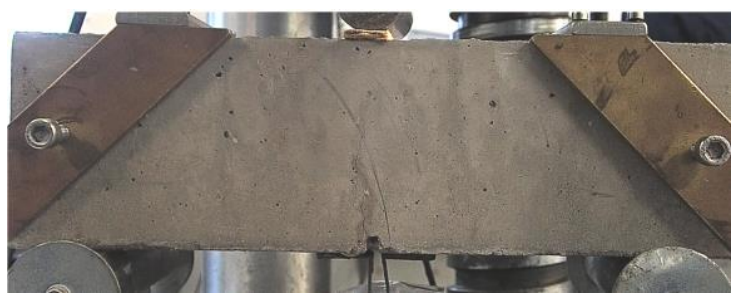

Fig. 1. Notched concrete beam tests with embedded SH

(ii) Uniaxial tension tests, which involved forming a discrete crack in a doubly notched specimen and subsequently supplying healing agent to the crack. The healing agent was then allowed to cure. In this experimental series, multiple tests were conducted with different fixed crack-opening displacements and different curing periods.

(iii) Dynamic flow tests, which measured the variation of the capillary contact angle with flow velocity for the selected healing agent (See Fig. 2). 


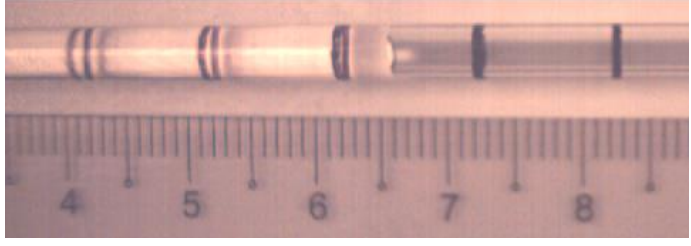

Fig. 2. Healing agent dynamic flow test

(iv) Curing front tests, in which the curing rate of healing agent was measured in a shallow 'bath' of agent adjacent to a concrete substrate (See Fig. 3).

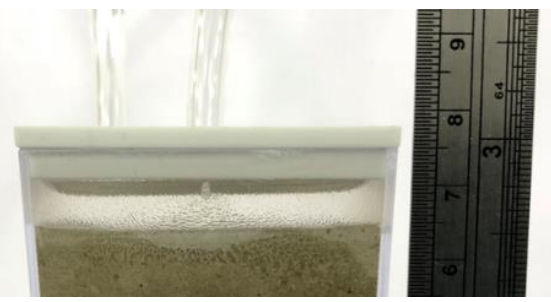

Fig. 3. Cyanoacrylate curing with a concrete substrate

(v) Sorption tests that involved measuring the flow of healing agent into a concrete sample through a natural cracked surface (See Fig. 4).

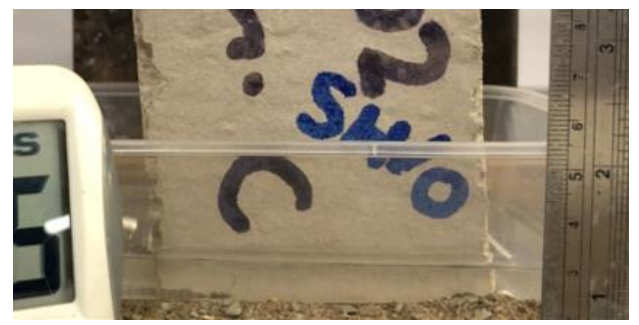

Fig. 4. Concrete specimen with a natural crack face in a bath of cyanoacrylate

An illustrative graph from a test on a $255 \times 75 \times 75 \mathrm{~mm}$ concrete beam (see Fig. 8), which had a central $5 \mathrm{~mm}$ notch and embedded channels for supplying healing agent, is given in Fig. 5. In the test shown, the healing agent was pressurised to 0.1 bar above atmospheric pressure. The mechanical load was applied such that the Crack Mouth Opening Displacement (CMOD), measured with a clip gauge, increased at a rate of $0.0002 \mathrm{~mm} / \mathrm{s}$.

It is evident from this test that the damage and healing processes were concurrent after the crack had formed. The results also include a control test in which no healing agent was supplied to the channels.

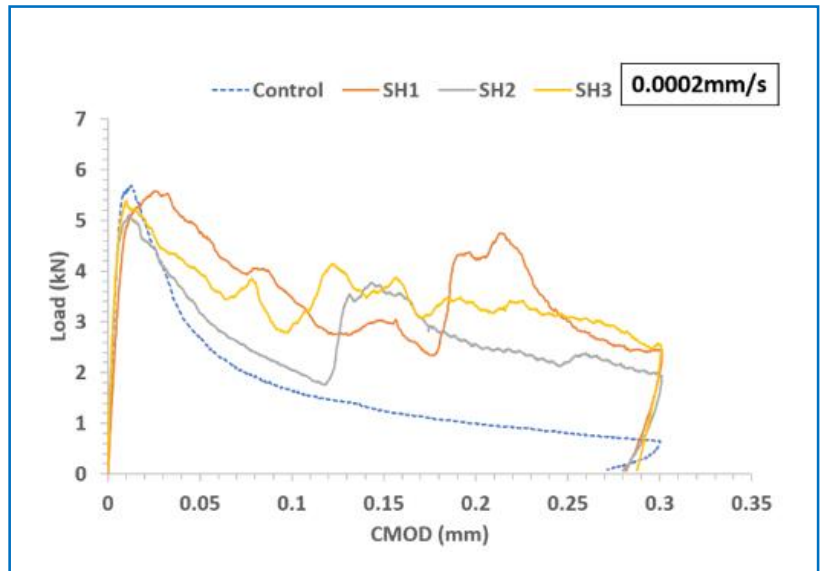

Fig. 5. Illustrative results from a damage-healing test.

\section{Numerical model}

The coupled numerical model includes components for simulating the flow of healing agent in discrete cracks, the transport of healing agent through a micro-cracked (and uncracked) concrete matrix, healing agent curing, cracking and healing. The key components of the model are now summarised.

\subsection{Capillary flow model}

Capillary flow in discrete cracks is simulated using the model proposed by Gardner et al [3], which is based on a modified Lucas-Washburn equation. The method has been previously validated against experimental data for a range of healing agents. The flow rate at the meniscus is given by equation (1);

$\dot{z}=\left(p_{c 0}\left(1-\beta_{s}\right)-\rho g z \sin (\varphi)\right) /\left(\frac{2 \beta_{m}}{b(z)}+\eta\right)$

where $\eta=A(z) \int_{0}^{z} 1 /\left(\frac{b(x) \beta_{w}}{2}+\frac{k(x)}{\mu}\right) A(x) d x$

and in which the superior dot denotes the time derivative, $z$ is the capillary rise height; $b(x)$ is the channel (crack) opening; $\beta_{w}, \beta_{s}$ and $\beta_{m}$ are the wall, stick-slip and meniscus correction parameters respectively; $\varphi$ is the capillary inclination angle; $\rho$ is the density of liquid; $g$ Is the gravitational acceleration; $\mu$ is the dynamic viscosity; $t$ is the capillary rise time $(s)$; the effective permeability term $k=b^{2} / 12$, and $\alpha$ is the inclination of the capillary wall.

An example set of validation data for the flow of healing agents in natural cracks in concrete specimens is given in Fig. 6 [3]. 


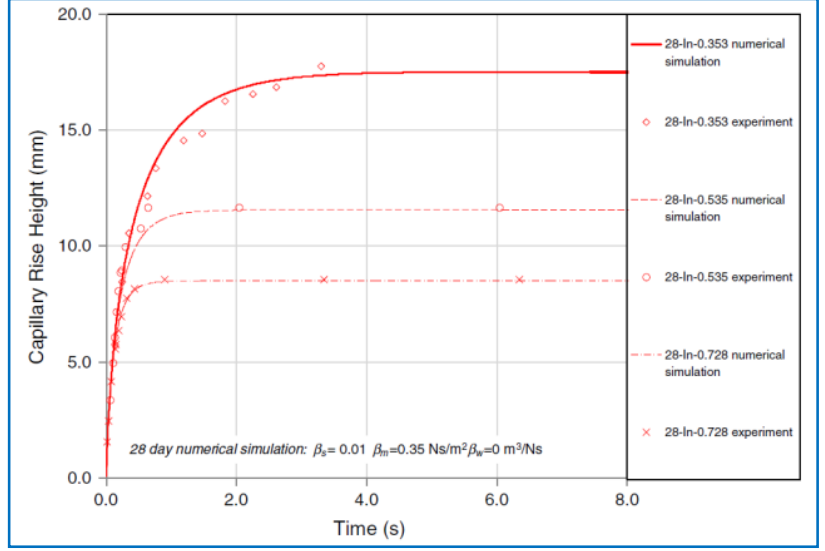

Fig. 6. Capillary rise height data at 28 days for inclined planar crack configuration.

\subsection{Damage-healing model}

The damage-healing part of the model is applied to a 'crack-plane', which is defined as the mid-surface of a narrow band of material that contains a macro-crack or an array of micro-cracks. The model relates the crackplane traction vector ( $\tilde{\boldsymbol{\tau}})$ to the relative displacement vector $(\tilde{\mathbf{u}})$, for which the damage-healing constitutive relationship is as follows:

$$
\tilde{\boldsymbol{\tau}}=(1-\omega) \tilde{\mathbf{k}}^{e} \overrightarrow{\mathbf{u}}+h \tilde{\mathbf{k}}^{h}\left(\tilde{\mathbf{u}}-\tilde{\mathbf{u}}_{h}\right)
$$

in which $\omega \in[0,1]$ is the damage parameter, $h \in[0, \omega]$ is the proportion of healed material and $\tilde{\mathbf{u}}_{h}$ is the permanent healing relative-displacement that ensures that the healing material forms in a stress-free state. $\tilde{\mathbf{k}}^{e}$ and $\tilde{\mathbf{k}}^{h}$ are elastic constitutive matrices for the original and healed material respectively. The inelastic component of $\tilde{\mathbf{u}}$ is computed from equation (3).

$$
\breve{\mathbf{u}}=\tilde{\mathbf{u}}-\left(\tilde{\mathbf{k}}^{e}\right)^{-1} \tilde{\boldsymbol{\tau}}
$$

The crack opening at any position is assumed to be equal to the normal component of $\breve{\mathbf{u}}$, which is denoted by the scalar $u_{f .}$.

The cohesive zone model has been applied to a 4noded element with embedded strong discontinuity within a research finite element program developed at Cardiff University.

\subsection{Healing agent curing}

The curing of cyanoacrylate can be simulated using the following curing function $(\phi)$;

$$
\phi\left(t, t_{0}\right)=1-e^{\frac{-\left\langle t-t_{0}\right\rangle}{\tau_{h}}}
$$

in which $t$ and $t_{0}$ represent the current and initial time respectively, and $\tau_{h}$ is a healing time parameters. $\langle>$ is a McCauley bracket, defined as $\langle x\rangle=0$ if $x \leq 0$ and $=x$ if $x>0$.
A comparison between equation (4) and some experimental data measured by the Cambridge Polymer group (2004) for the curing of a droplet of cyanoacrylate on glass is shown in Fig. 7.

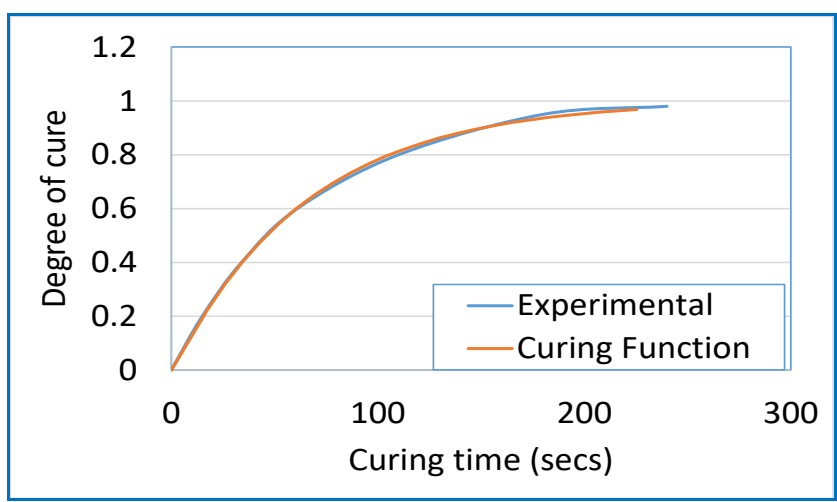

Fig. 7. Comparison between curing function (4) and experimental data from reference Cambridge Polymer Group (2004)

The volume of cured material $\left(v_{\text {cured }}\right)$ is obtained from the following convolution integral;

$v_{\text {cured }}=\int_{t_{0}}^{t} \phi\left(s, t_{0}\right) \frac{d v}{d s} d s$

During a time increment, new healing material may arrive, healed material may damage and damaged material may re-heal. To account for these factors the relative area of curing and cured material $(a)$ at a particular point is given by;

$a=a+\Delta a_{c}-\Delta a_{\text {redam }}+\Delta a_{\text {rec }}$

in which $\Delta a_{c}$ is the incremental area of virgin filled crack, $\Delta a_{\text {redam }}$ is the incremental area of re-damaged material and $\Delta a_{\text {rec }}$ is the incremental area of re-filled cracks;

The total relative proportion of healed material is then computed from equation (7).

$h=h \cdot e^{-\Delta t / \tau_{h}}+a \cdot\left(1-e^{-\Delta t / \tau_{h}}\right)$

This expression takes advantage of the semigroup property of the exponential function (equation 4) [4].

A much fuller description of the model, including full details of the damage-healing model, a description of the element with strong discontinuity, the diffuse surface curing model, the method used to track the increments of material arriving and curing, the matrix flow component and the computational details of the coupled non-linear solution procedure will be provided in a forthcoming journal paper on the model.

\section{Example}

The coupled model has been used to consider the set of experiments described in Section 2(i) of this paper. A particular example is presented here for illustration. Many more examples are presented in the forthcoming journal paper. 
The test setup is illustrated in Fig. 8. The beam was loaded such that the crack mouth opening displacement (CMOD) increased at a rate of $0.001 \mathrm{~mm} / \mathrm{s}$. The healing agent was supplied throughout the duration of the test. In addition, a control test was undertaken in which no healing agent was supplied.

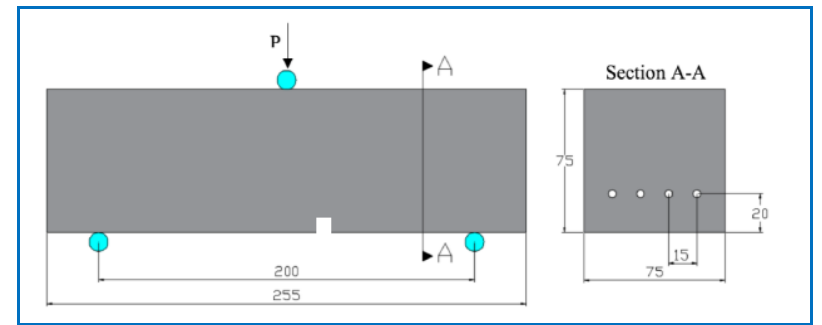

Fig. 8. Experimental arrangement

The component of the deformed mesh around the central crack is shown in Fig. 9. This shows the extent of healing at a time of 97 seconds from the start of the test.

A comparison between the model predictions and experimental data is provided in Fig. 10, in which Th and Th3 denote experimental results from healing tests. It may be seen that the coupled model provides a reasonable representation of the experimental behaviour.

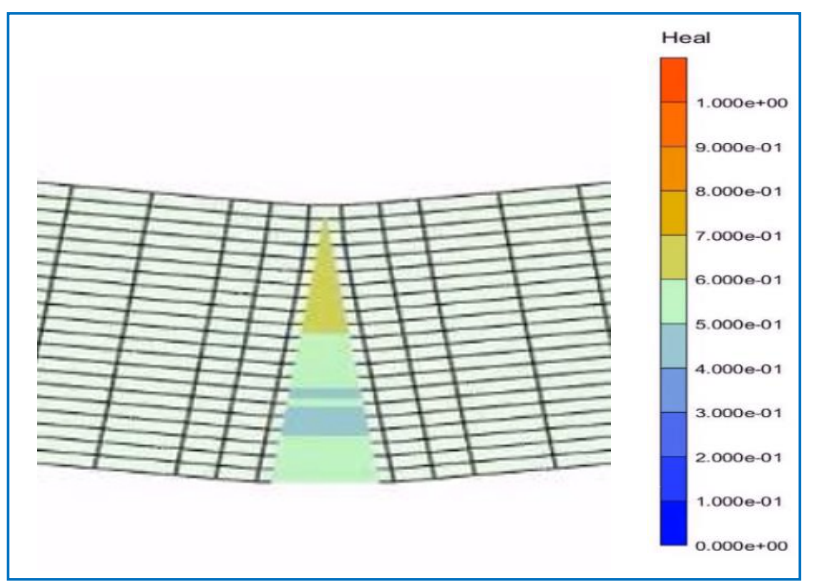

Fig. 9. Finite element mesh in deformed configuration with degree of healing

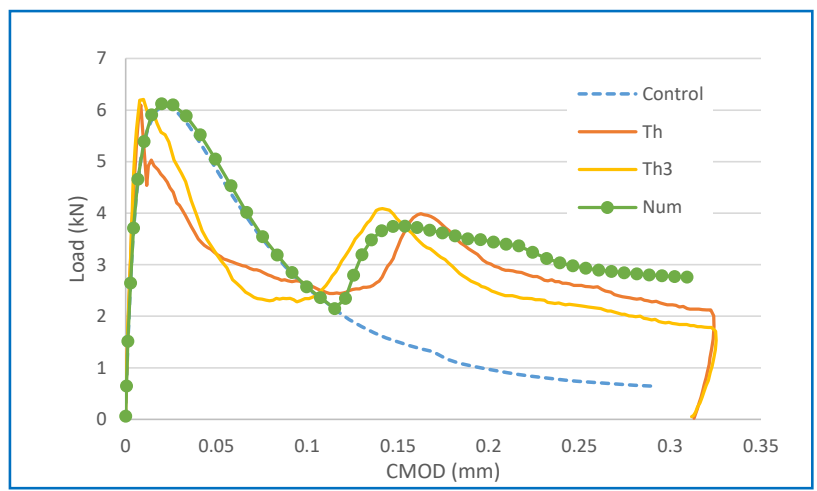

Fig. 10. Comparison between experimental and numerical results.

\section{Concluding remarks}

The data gathered in the experimental programme characterised the transport and curing properties of a healing agent in cracked cementitious structural elements. The tests also provided detailed information on damage-healing behaviour and shows how these processes interact when they occur simultaneously.

The coupled finite element model is able to represent the flow behaviour of a healing agent in a discrete crack and in a micro-cracked continuum. The model is also able to reproduce complex damage-healing behaviour with good accuracy.

\section{References}

1. N. De Belie at al. Review of Self-healing Concrete for Damage Management of Structures. Advanced Materials Interfaces. DOI: 10.1002/201800074

2. A. Jefferson, E. Javierre, B. Freeman, A. Zaoui, E.A.B. Koenders, and L. Ferrara.. Research Progress on Numerical Models for Self-Healing Cementitious Materials. Advanced materials interfaces, DOI: 10.1002/admi.201701378, (2018).

3. D.R. Gardner, A.D. Jefferson, A. Hoffman, R.J. Lark. Simulation of the capillary flow of an autonomic healing agent in discrete cracks in cementitious materials, Cement and Concrete Research , 58, (2014).

4. J. Mergheim, P. Steinmann. Phenomenological Modelling of Self-Healing Polymers Based on Integrated Healing Agents. Computational Mechanics 52 (3), 681-92, (2013).

\section{Acknowledgement}

The authors gratefully acknowledge the financial support from the UK Engineering and Physical Sciences Research Council (EPSRC) research grant EP/P02081X/1, Resilient Materials for Life (http://RM4L.com/). 